\title{
REVUE
}

\section{L'ORGANISATION ET L'EFFICIENCE DANS L'INDUSTRIE LAITIËRE}

\author{
par \\ G. GENIN \\ Ingénieur E.P.C.
}

\section{Le paiement des livraisons de lait aux fermiers}

Parmi les suggestions que l'on a proposées pour résoudre le problème de l'utilisation des excédents de production de beurre, il faut citer les efforts réalisés en vue d'assurer une meilleure utilisation des autres constituants du lait, comme par exemple lactose et protéines. Si ces produits en effet trouvaient des débouchés rémunérateurs, cela permettrait de diminuer parallèlement le prix de la matière grasse du beurre, peut-être de faciliter l'utilisation de cette matière grasse et la réduction du prix du beurre résultant de ces mesures permettrait à ce produit de lutter plus efficacement contre son concurrent direct : la margarine.

Une telle solution nécessiterait donc deux modifications, l'une portant sur les méthodes d'utilisation du lait, l'autre portant sur le mode de rétribution des fermiers fournisseurs de lait. Actuellement en effet, le lait, dans la plupart des pays producteurs, est payé aux fermiers d'après sa teneur en matière grasse. Un prix est fixé pour une teneur standard en matière grasse et des modifications sont faites suivant que le lait est plus ou moins riche en graisse.

Aux Etats-Unis, où la question de l'utilisation des surplus de beurre se pose plus particulièrement, on a envisagé d'autres méthodes de rétribution des fermiers. L'une de celles-ci consiste à établir le prix du lait d'après le prix de vente des substances extraites de ce lait, compte tenu d'une certaine marge couvrant les frais de traitement. Une autre méthode consiste à établir le prix d'achat du lait, d'après la valeur calorifique des produits alimentaires qu'il contient.

Plus récemment, E. O. Whitrier du Bureau of Dairy Industry, du Ministère de l'Agriculture des Etats-Unis, a proposé un autre procédé. Il estime en effet qu'il n'est pas pratique d'établir le prix d'achat du lait connaissant d'une part les proportions de graisse, de protéine et de sucre qu'il renferme et d'autre part le cours de ces produits d'après la valeur des autres aliments qui en renferment. Par exemple, il est très difficile d'établir une relation directe entre la valeur des protéines du lait et celle des protéines contenues dans 
les œufs, le bœuf, le porc, etc... D'autre part, il n'existe pas de méthode simple et rapide permettant de déterminer la teneur en sucre et en protéine du lait.

Par contre, on peut déterminer facilement la teneur en graisse du lait, ainsi que son résidu fixe, et il serait donc possible, partant d'un prix de base pour le lait standard, d'apporter à cette valeur quelques corrections pour tenir compte de sa teneur en graisse réelle et de sa teneur en résidu fixe. Comme la teneur en protéine du lait est à peu près constante dans le résidu fixe et égale à $26,5 \%$, cela équivaudrait à établir le prix du lait d'après sa teneur en graisse et en protéine et à considérer comme négligeables les différences dans les teneurs en sucre de lait. Cette façon de faire est d'autant plus justifiée que le prix du sucre de canne est relativement faible et qu'en reliant le prix du lactose au prix du sucre alimentaire, les erreurs seraient très faibles.

Dans ces conditions, Whitrier propose d'établir le prix du lait en appliquant la formule suivante :

$$
\mathbf{P}=\mathrm{Af}+\mathrm{Bs}
$$

dans laquelle $f$ est le nombre de kilogrammes de graisse contenus dans 100 kilogrammes de lait, A la valeur de la graisse au kilogramme, s le nombre de kilogrammes de résidu sec contenu dans 100 kilogrammes de lait et B la valeur accordée à ce résidu sec.

Dans le tableau ci-dessous, nous avons indiqué, en unités américaines, les prix de laits de différentes compositions, calculés d'après cette formule. Les prix indiqués dans ce tableau ont été établis soit en supposant qu'ils étaient uniquement calculés d'après la teneur en graisse du lait, ou au contraire établis en tenant compte à la fois de la teneur en graisse et de la teneur en résidu sec, et en supposant pour les prix de ces deux substances : graisse et résidu sec, des rapports différents.

Dans ce tableau, on a admis de donner la même valeur, dans tous les modes de calcul, à un lait contenant 3,5\% de graisse et $12,14 \%$ de résidu sec. On voit que si la valeur des protéines augmente

$\begin{array}{cccccc}\begin{array}{c}\text { Matière } \\ \text { grasse } \\ \text { en } \%\end{array} & \begin{array}{c}\text { Résidu sec } \\ \text { en } \%\end{array} & \begin{array}{c}\text { d'après la } \\ \text { teneur en } \\ \text { graisse }\end{array} & \begin{array}{c}\text { pour } \\ \mathrm{A}=2 \mathrm{~B}^{2}\end{array} & \begin{array}{c}\text { pour } \\ \mathrm{A}=\mathrm{B}^{\mathrm{s}}\end{array} & \begin{array}{c}\text { pour } \\ 2 \mathrm{~A}=\mathrm{B}^{4}\end{array} \\ 3,0 & 11,45 & 4,60 & 4,55 & 4,62 & 4,66 \\ 3,5 & 12,14 & 5,00 & 5,00 & 5,00 & 5,00 \\ 4,0 & 13,00 & 5,40 & 5.48 & 5,44 & 5.40 \\ 4,5 & 13,73 & 5,80 & 5,93 & 5,83 & 5,75 \\ 5,0 & 14,50 & 6,20 & 6,39 & 6,24 & 6,12 \\ 5,5 & 15,13 & 6,60 & 6,82 & 6,60 & 6,44 \\ 6,0 & 15,76 & 7,00 & 7,24 & 6,96 & 6,75\end{array}$


et au contraire la valeur de la graisse diminue, le prix d'un lait à faible teneur en graisse tend à augmenter et celui d'un lait à haute teneur en graisse tend à diminuer. Cependant, comme un lait à faible teneur en graisse est également à faible teneur en résidu sec, les différences tendent à se minimiser.

\section{Le nettoyage des tuyauteries dans les installations laitières}

Le nettoyage des différents appareils d'une laiterie répond à divers objets. Il faut par cette opération émulsifier les graisses qui se sont déposées dans les appareils, ramollir les protéines, neutraliser l'acidité du lait et empêcher la formation et le dépôt de pellicule sur les parois des appareils et les tuyauteries, dépôt qui résulte de la présence de composés minéraux dans le lait, les produits utilisés pour le lavage, l'eau de rinçage. Il faut ensuite procéder à un traitement de désinfection qui n'est plus une stérilisation absolue, mais qui a pour but de détruire la majorité des germes existant dans les appareils.

Aux Etats-Unis, d'après les règlements en vigueur publiés par une ordonnance du ministère de la Santé publique en 1939, le nettoyage des tuyauteries doit s'effectuer après démontage de celles-ci et le traitement de désinfection doit s'effectuer après remontage.

Or le démontage, le lavage et le remontage des tuyauteries est une opération qui demande quatre fois plus de temps qu'un nettoyage que l'on pourrait effectuer sans démontage. D'autre part, cette opération est considérée par le personnel comme particulièrement désagréable, elle est généralement effectuée par des manœuvres dont les salaires sont très bas et l'opération risque souvent d'entraîner une détérioration du matériel au cours des démontages et remontages successifs.

C'est la raison pour laquelle d'importants travaux ont été entrepris par différentes stations expérimentales afin d'établir la possibilité de procéder à ce nettoyage sans démontage des tuyauteries. Différents systèmes ont été proposés et par exemple, voici un procédé adopté dans une laiterie de Portland dans laquelle se trouvent 350 mètres environ de tuyauteries en acier stainless, de 50 à 60 millimètres de diamètre et certains raccords ou certaines parties de la tuyauterie exécutés en verre.

Le procédé conseillé pour le nettoyage de ces tuyauteries consiste à effectuer les opérations suivantes :

10 Pré-rinçage des tuyauteries par circulation d'eau fraîche portée à la température d'environ $40^{\circ}$ pendant 5 minutes; 
20 Lavage des tuyauteries en faisant séjourner une solution d'un agent détersif alcalin maintenu à une température de 55 à $75^{\circ}$ pendant 20 à 30 minutes;

$3^{\circ}$ Démontage des vannes et robinets qui sont lavés avec une solution tiède d'agent détersif, puis trempés dans une solution diluée d'un composé d'ammonium quaternaire;

$4^{\circ}$ Remontage des accessoires et rinçage de la tuyauterie avec de l'eau à une température de $50^{\circ}$ environ pendant 10 minutes ;

$5^{\circ}$ Passage dans la tuyauterie, pendant 10 minutes, d'une solution à 200 parties par million d'un hypochlorite.

On peut d'ailleurs modifier les conditions opératoires' et en particulier prévoir un lavage par une solution acide, suivi d'un rinçage à l'eau, avant le lavage par une solution d'un agent détersif alcalin.

Cette façon de faire permet de réduire très sensiblement les dépenses de nettoyage. Pour procéder au nettoyage après démontage des 350 mètres de tuyauterie de l'usine en question, il faut au moins 12 heures de travail chaque jour et l'assemblage et le remontage demandent à eux seuls plus de 6 heures de travail. Très souvent, il faut en outre procéder au changement des joints, de l'installation. Au contraire, si le nettoyage se fait sans démontage, par simple circulation, il ne faut plus que 3 heures de travail à peine, les opérations comportant non seulement le nettoyage proprement dit, mais également la préparation des solutions et toutes les liaisons nécessaires avec le réservoir de produits de nettoyage.

L'opération d'autre part évite de nombreuses détériorations au matériel, demande moins de fatigue au personnel et celui-ci travaillant avec plus de goût effectue un travail plus soigné. Il est donc probable que les règlements actuellement en vigueur vont être modifiés, afin d'autoriser ce mode de nettoyage des tuyauteries, à condition que l'opération soit faite avec les quantités de produit convenables.

\section{L'application des méthodes psychologiques en technologie laitière}

Dans toute industrie où on fait appel à la main-d'œurre, la psychologie peut jouer un rôle important, en améliorant les conditions de travail de l'ouvrier et en perfectionnant son habileté professionnelle. L,'industrie laitière est aujourd'hui relativement mécanisée, cependant elle utilise encore une main-d'œurre importante. C'est pour cela que le National Institut of Industrial Psychology de Grande Bretagne a procédé à des recherches portant 
sur l'emploi de la main-d'œuvre dans les laiteries et dans les fermes laitières.

En dehors des travaux qui ont porté sur la traite et plus particulièrement sur la traite manuelle, d'autres recherches ont été entreprises sur des opérations plus mécanisées et par exemple sur la fabrication du fromage. On s'est efforcé en particulier d'améliorer les tests subjectifs auxquels on fait encore appel dans cette industrie, de façon à parvenir à une fabrication plus régulière.

Un exemple de l'importance de ces travaux est donné par l'examen des différents procédés permettant d'apprécier la consistance de la caillebotte, qualité particulièrement importante dans la fabrication du fromage. Il semble que dans ce cas, il soit très difficile de remplacer les tests subjectifs par des contrôles objectifs, car on n'a pas trouvé d'appareil permettant d'évaluer la consistance d'un fromage, comme on n'a pas trouvé non plus de moyen permettant de donner une valeur numérique à la saveur d'un échantillon de beurre.

Il semble done que dans certains cas, malgré leurs gros inconvénients, les tests subjectifs sont plus utiles que les contrôles objectifs. Certes ils font appel à un personnel particulièrement compétent, mais ils sont encore plus rapides et plus économiques que l'utilisation d'appareils délicats donnant des renseignements incomplets.

On s'est d'ailleurs efforcé de réduire les inconvénients des tests subjectifs et l'application des théories de la statistique à ces tests a permis d'en resserrer la précision. Des modes opératoires ont pu être établis qui permettent, en suivant une technique scientifiquement établie pour le contrôle subjectif de produits tels que le beurre ou le fromage, de réduire au minimum le facteur personnel et d'éviter les erreurs manifestes. Il n'en reste pas moins qu'une formation très complète du personnel chargé de ces tests est nécessaire, et on s'aperçoit alors que la qualité d'un dégustateur devient en quelque sorte une seconde nature et l'intéressé est le plus souvent incapable d'expliquer par quel mécanisme il établit son jugement. Ce n'est que par un long apprentissage qu'on peut entraîner une personne à devenir compétente pour apprécier telle ou telle qualité d'un produit, mais la psychologie peut aider considérablement l'entraînement des spécialistes.

\section{L'organisation de la manutention dans l'industrie laitière}

La recherche d'une meilleure efficience dans l'exploitation des usines, le manque de main-d'œuvre dans certaines industries, la hausse des salaires, ont conduit depuis longtemps les industriels à s'efforcer de réaliser des économies dans les diverses activités 
de leurs usines et il est probable que ces économies sont les plus faciles à réaliser dans le domaine des frais de manutention.

C'est dans ce but que la «British Electrical Development Association " a présenté un film démontrant les bénéfices que l'on peut retirer d'une mécanisation des méthodes de manutention. Ce film est destiné à être projeté non seulement devant les dirigeants industriels, mais également devant les ouvriers, les contremaîtres, car on retire de sa projection, l'impression que des méthodes de manutention modernes, non seulement permettent de réaliser des économies, mais entraînent également une diminution considérable de la fatigue du personnel.

En général, ces méthodes de manutention modernes font appel à l'emploi de chariots à moteur comportant le plus souvent une plateforme mobile et pouvant être élevée par l'intermédiaire d'un moteur électrique, ce qui permet d'empiler sur des plateformes individuelles les matières premières ou objets fabriqués à manipuler.

Une autre solution consiste à faire appel à des chaînes transporteuses qui distribuent dans les différents ateliers de l'usine les matériaux en cours de transformation. La revue Milk Plant Monthly, dans son numéro d'avril 1953, a décrit par exemple le schéma d'une installation de magasinage de caisses de bouteilles de lait, ainsi que l'organisation du service de livraison qui doit non seulement assurer le chargement des camions de livraison, mais également s'occuper de la réception des bouteilles de lait vide et du magasinage des caisses en attente de livraison.

Dans l'installation décrite dans cette revue, il est fait appel à deux principes essentiels : le transport des caisses au moyen de tapis transporteurs et la manutention en hauteur des caisses, de façon à réaliser leur empilage et à réduire l'encombrement des magasins. Le même dispositif utilisé pour l'empilage des caisses permet également de retirer les caisses -des piles en vue de leur envoi vers la cour de chargement des camions.

Une telle installation mécanisée est d'un prix d'établissement assez élevé, mais particulièrement dans les pays à salaires importants, elle est rapidement amortie et certains des principes utilisés dans le magasin et dans le département d'expédition peuvent également être retenus pour assurer des manutentions dans les autres parties de la laiterie.

\section{Les économies d'eau dans les laiteries}

Un des postes les plus importants dans les dépenses d'eau d'une laiterie est représenté par l'eau de refroidissement utilisée pour abaisser la température du lait à sa sortie des installations de pasteurisation. Le temps est passé où on utilisait pour cela l'eau de la 
distribution qui était ensuite rejetée à l'égoût, et les installations récentes utilisent toujours la même eau qui, à sa sortie des échangeurs de température, est refroidie par des dispositifs dont il existe aujourd'hui différents modèles.

On a vu apparaître récemment un nouvel appareil de réfrigération des eaux, dénommé condenseur à surface d'évaporation, dans lequel on crée le froid nécessaire à la réfrigération de l'eau par évaporation forcée d'une partie de cette eau, l'évaporation étant accélérée par circulation d'un courant d'air à la surface des évaporateurs. Grâce à la chaleur latente d'évaporation de l'eau, on peut, moyennant une perte minime d'eau, refroidir des quantités importantes de ce liquide.

Ces appareils sont évidemment plus coûteux que des simples évaporateurs atmosphériques. Ils nécessitent une certaine dépense de courant électrique pour assurer la rotation des ventilateurs. Ils doivent comporter des dispositifs de contrôle et de régulation permettant de commander l'installation en fonction de la température de l'eau, de la température de l'air atmosphérique et d'autres facteurs, mais les économies qu'ils permettent de réaliser dans les dépenses d'eau sont telles qu'elles compensent largement les frais d'amortissement et de service de ce matériel.

\section{BIBLIOGRAPHIE ANALYTIQUE}

\section{LES LIVRES}

Schulz (M. E.), - Haltbare Milch. Sterilmilch und Acilmilch. (Lait de conserve. Lait stérilisé et lait acide de conserve.) 1 livre illustré, relié toile de 196 pages. Editeur : Hans Carl, Nürnberg, 1954. Prix : D.M. 12,85 (cartonné : 10,80).

Le Professeur M. E. Schulz, le savant Directeur de l'Institut Chimique de l'Etablissement de Recherches et d'Essais de laiterie de Kiel, est bien connu de nos lecteurs. Il a écrit ce livre en collaboration avee $M . M$. Kay, Kley et Voss à l'occasion des réunions de l'Association Internationale de la laiterie d'Amalfi, d'octobre 1954. Le lait stérilisé constitue un problème international. Dans les pays à climats chauds, les quantités de lait stérilisé produites ou importées sont encore d'importance minime par rapport aux quantités de lait pasteurisé, de lait condensé sucré, de lait évaporé et de lait en poudre, qui sont consommées. Il est remarquable qu'aux Etats-Unis où existent des zones de climats très ehauds, la consommation du lait stérilisé soit peu importante, de même qu'au Mexique, alors qu'en Europe sa production a beaucoup augmenté. Dans quelques villes des régions industrielles d'Angleterre, de Belgique et de Hollande, la consommation du lait stérilisé dépasse celle du lait pasteurisé. Ce développement récent pose la question 